\title{
A time and a place for everything? Temporal processing views of hippocampal function with special reference to attention
}

\author{
PAUL R. SOLOMON \\ Williams College, Williamstown, Massachusetts 01267
}

\begin{abstract}
A number of recent studies have indicated that the hippocampus is involved in classical conditioning of the rabbit's nictitating membrane response. In this paper, I briefly review these studies and then argue that the data are best accounted for in terms of the temporal processing view of hippocampal function. I further argue, within the context of the temporal processing approach, that the hippocampus is involved in attentional processes which enable the organism to learn to ignore irrelevant stimuli.
\end{abstract}

It was nearly 400 years ago that Julius Caesar Arantius coined the term hippocampus: "In its length it extends toward the anterior part of the brain, and is provided with a flexuous figure of varying thickness. This recalls the image of hippocampus, that is, of a little sea horse"' (Lewis, 1923). Since Arantius' initial description, the hippocampus has received considerable attention from researchers in a variety of disciplines. However, although the anatomy, physiology, and pharmacology are beginning to be well understood, the functions of the structure remain obscure.

The important finding by Milner and her coworkers (see Milner, 1970, for a review) indicating that humans with bilateral hippocampal damage could remember events prior to the lesion but had great difficulty recalling postlesion events has prompted a great deal of research aimed at replicating this deficit in animals (see Iverson, 1976). Despite numerous experiments which have examined the role of the hippocampus in a variety of learning paradigms, there is as yet no viable animal analogue of the clinical condition. Nevertheless, this work has led to a good deal of speculation as to how the hippocampus might participate in the learning process.

Recently, two apparently competing theories of hippocampal function have emerged: the spatial mapping view and the temporal processing view. The

Some of the research described in this paper was supported by grants from the National Science Foundation (BNS-77-14871), the National Institute of Mental Health (MH-33381-01), and a Faculty Research Grant from Williams College. Parts of this paper were prepared while the author was an NIMH postdoctoral fellow (MH08105-01) in R. F. Thompson's laboratory at the University of California, Irvine. I am grateful to Karen Gottfried and Donna Staton for their helpful comments on an earlier version of the paper. spatial mapping hypothesis argues that the hippocampus acts as part of a neural system which forms a cognitive map of the environment. Central representations of separate places in the environment, as well as the relationship of one place to another, are then represented (see O'Keefe \& Nadel, 1978). The primary support for this view comes from electrophysiological recordings both from freely moving rats (O'Keefe \& Dostrovsky, 1971) and from rats required to solve problems in a radial arm (Olton, Branch, \& Best, 1978) or elevated T-maze (O'Keefe, 1976). These studies showed that the behavior of single cells in the hippocampus correlated with the animal's location in the environment. A second line of supporting evidence indicates that rats with fornical lesions (O'Keefe, Nadel, Kieghtly, \& Kill, 1975) as well as lesions of the hippocampus (O'Keefe \& Black, 1978) or its extrinsic fiber connections (Olton, Walker, \& Gage, 1978) were disrupted in their ability to solve problems which required spatial mapping.

The temporal processing view of hippocampal function argues that one function of the hippocampus is to register the temporal sequence of events (e.g., Berger \& Thompson, 1978a; Moore, 1979; Solomon, 1979). In classical conditioning, for example, this might simply mean the relationship between the CS and the UCS. It could, however, refer not only to the temporal sequence of stimuli, but also to the sequence of stimuli and responses (see Moore, 1978). It is important to note from the outset that a temporal processing view does not mean that the hippocampus could not also map the relationship between spatial events. Indeed, as Moore (1979) has argued, it may be very difficult to separate the space-time dimension. Similarly, Berger and Thompson (1978a) and Solomon (1979) have suggested that the type of coding that occurs in the hippocampus may be influenced by the type of problem the animal is asked to solve. 
The primary support for the temporal mapping viewpoint comes from studies of classical conditioning of the rabbit's nictitating membrane response (NMR). I have argued elsewhere (Solomon, 1979) that spatial cues play little, if any, role in this task. Nevertheless, data from electrophysiological (Berger, Alger, \& Thompson, 1976; Berger \& Thompson, 1978a; Berry \& Thompson, 1978; Thompson, Berger, Cegavske, Patterson, Roemer, Teyler, \& Young, 1976), lesion (Solomon, 1977; Solomon \& Moore, 1975; Buchanan, News, \& Powell, Note 1), and stimulation (Salafia, Chiaia, \& Ramirez, 1979; Salafia, Romano, Tynan, \& Host, 1977) studies, as well as some pharmacological work ongoing in our lab, indicate that the hippocampus is involved in conditioning of the rabbit's NMR.

In this paper, I will briefly review the evidence suggesting hippocampal involvement in classical conditioning of the rabbit's NMR. I will then argue that these data are best accounted for in terms of a temporal processing view of hippocampal function. I will also argue, within the context of the temporal processing approach, that the hippocampus is involved in attentional processes which enable the organism to learn to ignore irrelevant stimuli.

\section{The Hippocampus in Classical Conditioning of the Rabbit's NMR}

Electrophysiological studies. The first indication that neural activity in the hippocampus was correlated with conditioning of the rabbit's NMR came from a study by Berger et al. (1976). The authors reported an increase in activity in the pyramidal and granule cell layers of the dorsal hippocampus which was highly correlated with the conditioned NMR. This increased hippocampal activity, which preceded the behavioral response by $35-40 \mathrm{msec}$, began during the first eight CS-UCS pairings. Initially, the hippocampal response preceded the UCR, but, as CRs began to occur, the neural response moved forward in the interstimulus interval, always preceding the behavioral response by $35-40$ msec.

In an attempt to relate hippocampal and septal unit activity, Berger and Thompson (1978b) recorded simultaneously from the medial or lateral septum and the hippocampus during NMR conditioning. They found that unit activity in the lateral septum essentially paralleled that of the hippocampus, whereas activity in the medial septum did not seem to be linked to CSUCS pairings, but rather was correlated with the tone CS and the airpuff UCS presentations. Based on these data, Berger and Thompson (1978a, 1978b) suggested that the change in neural activity in the hippocampus and lateral septum might be one of the earliest neuronal indicators that learning has occurred.

Additional evidence for the role of the septohippocampal system in conditioning of the rabbit's NMR comes from a recent study by Berry and
Thompson (1978). In this study, the authors were able to predict the rate of acquisition of the NMR by examining hippocampal EEG immediately prior to conditioning. Rabbits which displayed relatively high levels of activity in the low-frequency range $(2-8 \mathrm{~Hz})$ conditioned significantly faster than animals which had a preponderance of activity in the high-frequency range $(8-22 \mathrm{~Hz})$.

Lesion studies. The work in our lab (Solomon, 1977; Solomon \& Moore, 1975) is consistent with the work of others (Schmaltz \& Theios, 1972; Buchanan et al., Note 1) in demonstrating that hippocampal lesions do not affect acquisition of the rabbit's NMR. But these data do indicate that hippocampal lesions disrupt the animal's ability to learn to ignore irrelevant stimuli. This interpretation is based primarily on studies of latent inhibition and blocking of the rabbit's NMR.

Latent inhibition refers to the finding that a series of nonreinforced preexposures to a stimulus retards conditioning to that stimulus when it is subsequently paired with a UCS or reinforcing event (see Lubow, 1973, for a review). Investigations of this phenomenon in the rabbit's NMR preparation (Reiss \& Wagner, 1972; Solomon, Brennan, \& Moore, 1974) agree with data from other preparations (CER, Rescorla, 1971; two-way avoidance, Solomon, Kiney, \& Scott, 1978) in indicating that this retardation of conditioning is due to the preexposed stimulus losing salience via a habituation-like process whereby the animal learns to ignore the irrelevant (preexposed) stimulus (see Mackintosh, 1975).

In one study conducted on the LI effect, we (Solomon \& Moore, 1975) reported that whereas 450 tone preexposures retarded conditioning in normal rabbits and rabbits with cortical lesions, animals with hippocampal lesions showed no such effect. Our interpretation of these data was that hippocampal lesions disrupted the animal's ability to learn to ignore an irrelevant CS. To further test this view of the role of the hippocampus, we examined the effects of dorsal hippocampal ablation in Kamin's two-stage blocking paradigm.

Blocking of the rabbit's NMR is typically carried out in a two-stage design (cf. Marchant \& Moore, 1973). In Stage 1, the blocking group is presented with a tone CS paired with an eyeshock UCS until the CR is well established. The control group simply sits in the apparatus for a corresponding amount of time. Stage 2 is the same for all animals and consists of acquisition of a compound CS consisting of the tone from Stage 1 plus a light. When both groups display a high level of conditioning to the compound, the test phase is introduced. During testing, all animals receive nonreinforced presentations of the tone interspersed with light presentations. In general, while animals in the control condition respond to both the light and 
tone during extinction, animals in the blocking group respond only to the tone.

Blocking, according to some views (Kamin, 1969; Mackintosh, 1975), is an attentional process whereby animals in the blocking group learn to ignore the redundant light in Stage 2, because it gives no new information about the UCS. Blocking then is similar to latent inhibition in that the animal must learn to ignore an irrelevant (redundant) stimulus. According to this view, hippocampal lesions should disrupt blocking. Solomon (1977) reported just this finding. Although normal rabbits and rabbits with cortical lesions responded only to the tone during testing, that is, they showed blocking, hippocampectomized rabbits responded to both the tone and light during the test phase.

Posttrial hippocampal stimulation. A series of studies by Salafia and his co-workers (Salafia, Chiaia, \& Ramirez, 1979; Salafia, Romano, et al., 1977) indicate that posttrial hippocampal stimulation immediately following the termination of the UCS retards acquisition of the behavioral CR. This appears to be the case whether or not the stimulation produces discharges. Once CRs begin to occur, however, posttrial stimulation has no effect. Based on these data, Salafia et al. (1977) concluded that posttrial stimulation disrupted the association between the CS and UCS.

Recently, Salafia and Allan (1980) reported that hippocampal stimulation following CS preexposure either retarded or enhanced the LI effect. Which of these effects hippocampal stimulation produced appeared to be affected by the number of CS preexposures and the parameters of the post-CS hippocampal stimulation. Although the relationship between hippocampal stimulation and LI remains obscure, Salafia (1980) has suggested that hippocampal stimulation works by altering the salience of the CS. This view parallels our (Solomon \& Moore, 1975) interpretation of the effects of hippocampal lesions on LI, and also fits nicely with Moore and Stickney's (1980) computer simulations, which indicate that the effects of hippocampal manipulations can be accounted for by their effects on the salience parameter of Mackintosh's (1975) conditioning model.

Pharmacological studies. Comparatively little is known about the pharmacological systems involved in conditioning the rabbit's NMR. But there are data to suggest cholinergic involvement, and we have recently collected some preliminary data to suggest that the septohippocampal cholinergic system is involved.

Moore, Goodell, and Solomon (1976) reported that systemic administration of the anticholinergic scopolamine disrupted conditioning but not terminal performance of the conditioned NMR. A similar finding was reported by Downs, Cardoza, Schneiderman, Yehle, Van Decar, and Zwilling (1972) following administration of atropine. These data, in conjunction with electrophysiological data indicating septohippo- campal involvement in conditioning and the anatomical data indicating a large septohippocampal cholinergic pathway in the rabbit (Dudar, 1975; Smith, 1974), suggest that the septohippocampal cholinergic system might be the locus of scopolamine-induced disruption of the conditioned NMR.

In a study currently being conducted in my lab by Karen Gottfried, we examined the effects of microinjections of scopolamine into the dorsal hippocampus or the medial septum on acquisition of the conditioned NMR. In the first of our studies, we found that daily microinjections of scopolamine into the hippocampus ( $1 \mu \mathrm{l}$ bilaterally, $20 \mu \mathrm{g} / \mu \mathrm{l}$ ) did not affect conditioning of the NMR to a light CS and an eyeshock UCS. Similar injections into the medial septum, however, significantly retarded CR acquisition (see Figure 1). Subsequent tests revealed that these differences were not due to drug-induced changes in CS or UCS sensitivities.

\section{Temporal Coding in the Hippocampus and the Attention Processes}

The data presented in the previous sections indicate that we have learned a good deal in a relatively short period of time about the role of the hippocampus in classical conditioning of the rabbit's NMR. But the results of these studies also raise several important questions. Perhaps the most fundamental of these involves the apparent conflict between the lesion and electrophysiological data.

The electrophysiological data clearly indicate that the intact hippocampus is involved in the conditioning process. Moreover, these data suggest that early in conditioning the activity of hippocampal units reflects a contingency between the CS and UCS. As Rescorla (1967) has pointed out, the CS-UCS contingency may

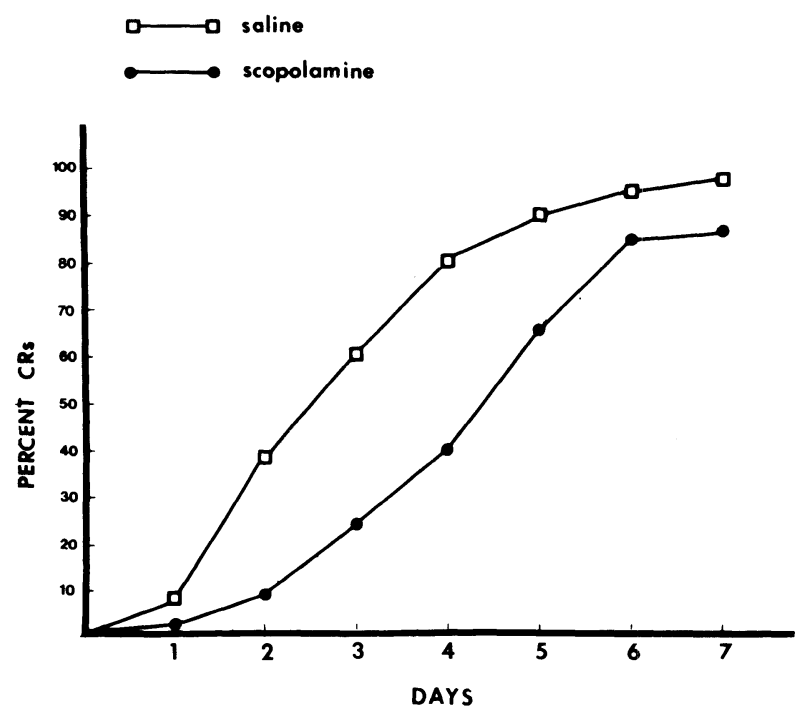

Figure 1. Mean percent CRs for rabbits in the scopolamine and saline groups over the 7 days of conditioning. 
be the critical factor in classical conditioning. The lesion data, however, indicate that rabbits with bilateral dorsal hippocampal ablation have no difficulty in acquisition of the conditioned NMR. Although these data seem to conflict, there are a number of possible explanations.

The finding that removal of a neural structure does not disrupt conditioning cannot be interpreted to mean that the structure does not participate in the behavior of the intact animal. Thus there is the possibility, as Thompson et al. (1976) have pointed out, that structures other than hippocampus (e.g., brainstem) may show similar changes in neural activity early in conditioning. It is possible, then, that the hippocampus is receiving information that is similar to that being received by other areas of the brain with regard to the temporal relationship between the CS and UCS but is using this information for different purposes. In this way, the hippocampus could be involved in aspects of conditioning other than the actual association of the CS and UCS.

It is likely that learning is a multistage process with different anatomical and physiological substrates for each stage (see Thompson et al., 1976). To take a simplified approach, execution of the conditioned response would at minimum require (a) attention to relevant stimuli and tuning out of irrelevant stimuli, (b) association of the CS and UCS, and (c) organization and execution of the motor response. Although the hippocampus has been implicated in both $a$ and $b$ (see Table 1), it may be possible to account for the data involving the role of the hippocampus in classical conditioning of the NMR under the general heading of attentional processes and, more specifically, the role of the hippocampus in tuning out irrelevant stimuli.

We have argued elsewhere (Moore, 1979; Solomon, 1979) that the hippocampus is involved in the temporal processing of stimuli. This simply means coding of the temporal sequence of events. In the rabbit's NMR this would be the coding of the temporal relationship of the CS and the UCS or between the CS, CR, and UCS. The electrophysiological data indicate that neurons in the hippocampus are capable of this type of activity.

The temporal mapping view appears able to account for the disruption of LI and blocking in hippocampectomized rabbits. In LI, the hippocampus receives information that a stimulus (CS) has been received, but this is not closely followed in time by a motivationally significant event (e.g., a UCS). Following this pattern of information, the hippocampus participates in a process whereby the irrelevant CS is tuned out. The effects of hippocampal lesions on conditioning are consistent with this view, and there is electrophysiological data in the rat indicating that hippocampal unit activity during conditioning is altered following CS preexposure (Best \& Best, 1976). Un- fortunately, hippocampal activity in the rabbit during LI has yet to be monitored.

In blocking, the animal learns a slightly more complex temporal sequence-that $\mathrm{CS}_{\mathrm{A}}$ always predicts the UCS, and that any additional information, e.g., $\mathrm{CS}_{\mathrm{B}}$, is redundant and should be tuned out. The lesion data indicate that the hippocampus is also involved in this process.

It is noteworthy that, in blocking, $\mathrm{CS}_{\mathrm{B}}$ is always paired with the UCS (i.e., there is contiguity) but is nevertheless tuned out in normal animals, and this process is disrupted in hippocampals. This suggests that the hippocampus is capable of coding more than contiguity between two stimuli, since, if it coded only contiguity, $\mathrm{CS}_{\mathrm{B}}$ should not be tuned out. Rather, as the electrophysiological data suggest, the hippocampus appears to recognize contingencies (and lack of contingencies) between CSs and UCSs.

The temporal processing or attentional view may also be capable of handling the electrophysiological data. During conditioning, the hippocampus receives information that the CS predicts the UCS, that is, there is a contingency. This contingency is indicated in the behavior of hippocampal units during CS-UCS pairings (Berger \& Thompson, 1978a). But, as indicated earlier, it is possible that this temporal coding is necessary for processes other than the actual association of the CS and the UCS. Specifically, this coding may be necessary for attentional processes. If the hippocampus is involved in tuning out irrelevant stimuli, CSs that do not uniquely predict the occurrence of the UCS, then it would need to code contingencies between the CS and UCS. Although it would code this information during excitatory conditioning, it would not have to be involved in tuning out the CS, since, in excitatory conditioning, the CS is relevant. Thus, although the pattern of firing of hippocampal neurons would reflect the temporal relationship of the CS and UCS during acquisition of the conditioned NMR, hippocampal lesions would not affect this process.

Put somewhat differently, when the hippocampus codes a temporal contingency between the CS and the UCS, it allows the organism to attend to the CS. But when the CS is irrelevant, the hippocampus acts as part of a neural system which allows the animal to cease attending to that stimulus. Since it would be most adaptive for an organism to learn that a particular stimulus was irrelevant after the first few presentations, the hippocampus should begin to code the temporal relationship of stimuli early in conditioning. Consistent with this is the finding that the electrophysiological changes in the hippocampus actually begin to occur by the second CS-UCS pairing (Berger \& Thompson, 1978a).

Implicit in the view that the hippocampus is involved in conditioning processes other than the association between the CS and UCS is that other structures 
Table 1

Research Indicating Septo-Hippocampal Involvement in the Basic Processes Necessary for Elaboration of the Conditioned NMR

Attentional Processes

Medial septal unit activity during conditioning (Berger \& Thompson, 1978b).

Prediction of learning rate by hippocampal EEG (Berry \& Thompson, 1978).

Failure of hippocampal lesions to disrupt NMR conditioning (Buchanan, News, \& Powell, Note 1; Schmaltz \& Theios, 1972; Solomon, 1977; Solomon \& Moore, 1975).

Disruption of latent inhibition following hippocampal lesions (Buchanan, News, \& Powell, Note 1; Solomon \& Moore, 1975).

Disruption of blocking following hippocampal lesions (Solomon, 1977).

Disruption of hippocampal RSA and conditioning following medial septal lesions (Berry \& Thompson, 1979). Impaired differential conditioning following septal lesions (Lockhart \& Moore, 1975; Powell, Milligan, \& Buchanan, 1976).

\section{$\leftarrow$}

Posttrial stimulation-induced disruption of latent inhibition (Salafia,1979).

Conditioning or enhanced UCS excitability with abducens stimulation as the UCS (Mis, Gormezano, \& Harvey, 1979;

Young, Cegavske, \& Thompson, 1976).

Systematically administered anticholinergicinduced disruption of conditioning (Downs, Cardoza, Schneiderman, Yehle, Van DeCar, \& Zwilling, 1972; Moore, Goodell, \& Solomon, 1976).

Disruption of conditioning following scopolamine injected into medial septum (Solomon \& Gottfried, 1979).

Note-Each study is placed under the process in which the research suggests the septum or hippocampus is involved. Arrows indicate that the study may be interpreted to indicate septohippocampal involvement in other processes, as described in the text. The studies that indicate motor aspects of the NMR are provided for reference purposes.

are capable of coding this relationship. There are now several studies to suggest that the midbrain may be involved in this process.

A recent study by Mis, Gormezano, and Harvey (1979) indicated that direct stimulation of the abducens nucleus could serve as a UCS for acquisition of the conditioned NMR. Similar results have been reported by Powell (1980), working in Moore's lab, and a study by Young, Cegavske, and Thompson (1976) showed that a tone paired with electrical stimulation of the abducens nucleus produced both increased amplitude of the UCR and some evidence of conditioning. As Mis et al. have indicated, these data suggest that the integration of information necessary for the elaboration of a conditioned response can occur in the brainstem.

It is also noteworthy that the midbrain seems capable of supporting inhibitory conditioning (Mis, 1977), and this fits nicely with work indicating that the hippocampus is not involved in conditioned 
inhibition of the rabbit's NMR (Solomon, 1977).

A second, but related, question regarding the role of the hippocampus in NMR conditioning is: Why are manipulations which seem to affect hippocampal activity more detrimental to conditioning than ablating the structure?

As indicated earlier, Berry and Thompson (1978) reported that the rate of NMR conditioning could be predicted by examining hippocampal EEG patterns immediately prior to CS-UCS pairings, with animals in the low-frequency range conditioning more quickly than those in the high-frequency range. To account for this, the authors suggested that hippocampal RSA might be an indication of a "behavioral state" that plays an important role in learning. Interestingly, manipulations which seem to disrupt this behavioral state, as indicated by an attenuation of hippocampal RSA, also seem to disrupt conditioning.

Systemic administration of scopolamine both blocks hippocampal RSA in the rabbit (Stumpf, Petsche, \& Gogolak, 1962) and disrupts conditioning of the rabbit's NMR (Moore et al., 1976). Similarly, data collected in my lab by Karen Gottfried indicate that microinjections of scopolamine into the medial septal nucleus, but not into the dorsal hippocampus, retard acquisition of the NMR. It will be important ultimately to determine if these microinjections also disrupt RSA, although this does seem to be the case in the rat (cf. Bennett, 1975). A recent study by Berry and Thompson (1979) parallels the pharmacological data by indicating that lesions limited to the medial septal nucleus which impaired hippocampal RSA also retarded conditioning of the NMR. This may be particularly important in view of previous data indicating that larger septal lesions had no effect on acquisition of the conditioned NMR (Lockhart \& Moore, 1975).

It appears, then, that disrupting hippocampal RSA by a variety of means also retards acquisition of the NMR. But how might this disruption of RSA or behavioral state be reflected in the hippocampus? Perhaps the disruption of RSA adversely affects the temporal coding of stimuli. This, in turn, could lead to a tuning out of all CSs, including the CS that uniquely predicts the occurrence of the UCS. In this way, activation of the hippocampus would actually be more detrimental to conditioning than would removal of the structure (Isaacson, 1972, has suggested a similar view). This view leads to an interesting set of predictions. For example, could it be that administration of scopolamine or medial septal lesions would not retard conditioning in hippocampectomized rabbits?

The data indicating a disruption of conditioning following posttrial stimulation (Salafia et al., 1977) also seem to be consistent with this view. It is possible that hippocampal stimulation, like disruption of RSA, could disrupt the animal's ability to code CS-UCS relationships, again resulting in a tuning out of all stimuli (see Moore, 1978). Of course, it will be difficult to determine if small medial septal lesions, scopolamine, and posttrial stimulation all affect the hippocampus in a similar way, but it is interesting to note that all of these manipulations have similar effects on acquisition of the CR. That is, they delay the onset of conditioning, but once CRs begin to occur, the rate of conditioning is the same as in control animals, and, like control animals, rabbits that receive these manipulations all reach asymptotic levels of conditioning. To place this in an existing theoretical framework for NMR conditioning, the manipulations seem to affect Phase 1 of Prokasy's (1972) two-stage conditioning model.

\section{Temporal vs. Spatial Information Processing in the Hippocampus?}

The data from the rabbit NMR preparation strongly implicate the hippocampus in the coding of temporal relationships. In this article, I have argued that the hippocampus may use this temporal information to allow the animal to ignore stimuli which do not uniquely predict a change in the probability of the occurrence of the UCS. This, in turn, allows the animal to attend to stimuli which do signal such change.

As we have indicated elsewhere (see Moore, 1979; Solomon, 1979), this does not preclude hippocampal involvement in the coding of spatial relationships (cf. O'Keefe \& Nadel, 1978). Indeed, it may be exceedingly difficult (and perhaps not warranted) to design experiments which will measure behavior guided by either purely spatial or purely temporal relationships. A more productive strategy may be to view the hippocampus as part of a neural system which is capable of encoding the relationship between many kinds of stimuli. What particular stimuli are coded could be a function of what type of relationship the animal is asked to learn. In the rabbit NMR preparation, these problems are primarily temporal in nature. In the radial arm maze, the problems are primarily spatial.

Clearly, the arguments can be made that there are spatial components to the rabbit NMR preparation (e.g., contextual cues which Nadel and Willner, 1980, equate with places; but see Berger, Clark, \& Thompson, 1980, for an alternative interpretation of context) and that there are temporal components to maze learning (see Moore, 1979). Similarly, it can be argued that the rabbit NMR preparation precludes an evaluation of spatial relationships or that maze learning biases the situation against evaluating the role of temporal relationships (see Berger \& Thompson, 1978a). But, in light of the evidence on hippocampal involvement in coding both temporal and spatial relationships, it may be difficult to argue that all of the data can be accounted for by one theory to the exclu- 
sion of the other. To do this would require either dismissing or radically reinterpreting one substantial line of research. Rather than attempting this, it may be more productive to recognize that different preparations provide means of examining different types of relationships among stimuli. It would then be possible to attempt to understand how the hippocampus (as well as other neural structures) codes this information and subsequently uses it to enable the animal to exhibit adaptive behavior.

\section{REFERENCE NOTES}

1. Buchanan, S., News, J., \& Powell, D. A. Autonomic-somatic relationships: Effects of hippocampal lesions on Pavlovian conditioning in the rabbit. Manuscript submitted for publication, 1979.

\section{REFERENCES}

Bennett, T. L. The electrical activity of the hippocampus and processes of attention. In R. L. Isaacson \& K. H. Pribram (Eds.), The hippocampus (Vol. 2). New York: Plenum Press, 1975.

Berger, T. W., Alger, B. E., \& Thompson, R. F. Neuronal substrates of classical conditioning in the hippocampus. Science, 1976, 192, 483-485.

Berger, T. W., Clark, G. A., \& Thompson, R. F. Learningdependent neuronal responses recorded from limbic system brain structures during classical conditioning. Physiological Psychology, 1980, 8, 155-167.

Berger, T. W., \& THOMPSON, R. F. Limbic system interrelations: Functional division among hippocampal-septal connections. Science, 1977, 197, 587-589.

Berger, T. W., \& Thompson, R. F. Neuronal plasticity in the limbic system during classical conditioning of the rabbit nictitating membrane response. I. The hippocampus. Brain $R e$ search, 1978, 145, 323-346. (a)

Berger, T. W., \& Thompson, R. F. Neuronal plasticity in the limbic system during classical conditioning of the rabbit nictitating membrane response. II. Septum and mammillary bodies. Brain Research, 1978, 156, 293-314. (b)

Berry, S. D., \& Thompson, R. F. Prediction of learning rate from hippocampal EEG. Science, 1978, 200, 1298-1300.

Berry, S. D., \& Thompson, R. F. Medial septal lesions retard classical conditioning of the nictitating membrane response of rabbits. Science, 1979, 205, 209-210.

Best, M. R., \& Best, P. J. The effects of state of consciousness on latent inhibition in hippocampal unit activity in the rat during conditioning. Experimental Neurology, 1976, 51, 564-573.

Cegavske, C. F., Thompson, R. F., Patterson, M. M., \& Gormezano, I. Mechanisms of efferent control of the reflex nictitating membrane response in rabbit (Oryctolagus cuniculus). Journal of Comparative and Physiological Psychology, 1976, 90, 411-423.

Downs, D., Cardoza, C., Schneiderman, N., Yehle, A. L., Van Decar, D. H., \& Zwilling, G. Central effects of atropine upon aversive classical conditioning in rabbits. Psychopharmacologia, 1972, 23, 319-333.

DudAR, J. D. The effect of septal nuclei stimulation on the release of acetylcholine from rabbit hippocampus. Brain Research, 1975, 83, 123-133.

IsAACSON, R. L. Neural systems of the limbic brain and behavioral inhibition. In R. A. Boakes \& M. S. Halliday (Eds.), Inhibition and learning. New York: Academic Press, 1972.
Iverson, S. D. Do hippocampal lesions produce amnesia in animals? In C. C. Pfeiffer \& J. R. Smythies (Eds.), International review of neurobiology. New York: Academic Press, 1976.

Kamin, L. J. Predictability, surprise, attention and conditioning. In B. A. Campbell \& R. M. Church (Eds.), Punishment and aversive behavior. New York: Appleton-Century-Crofts, 1969.

LEWIS, F. T. The significance of the term hippocampus. Journal of Comparative Neurology, 1923, 35, 213-230.

LockharT, M., \& Moore, J. W. Classical differential and operant conditioning in rabbits (Oryctolagus cuniculus) with septal lesions. Journal of Comparative and Physiological Psychology, $1975,88,147-154$.

Lubow, R. E. Latent inhibition. Psychological Bulletin, 1973, 79, 398-407.

Mackintosh, N. J. A theory of attention: Variations in the associability of stimuli with reinforcement. Psychological Review, 1975, 82, 276-298.

Marchant, H. G., \& Moore, J. W. Blocking on the rabbit's conditioned nictitating membrane response in Kamin's twostage paradigm. Journal of Experimental Psychology, 1973, 101, 155-158.

Milne R, B. Memory and medial temporal regions of the brain. In K. H. Pribram \& D. E. Broadbent (Eds.), Biological basis of memory. New York: Academic Press, 1970.

Mis, F. L. A midbrain-brainstem circuit for conditioned inhibition of the rabbit's (Oryctolagus cuniculus) nictitating membrane response. Journal of Comparative and Physiological Psychology, 1977, 91, 975-980.

Mis, F. W., Gormezano, I., \& Harvey, J. A. Stimulation of abducens nucleus supports classical conditioning of the nictitating membrane response. Science, 1979, 206, 473-475.

MOORE, J. W. Brain processes and conditioning. In A. Dickinson \& R. A. Boakes (Eds.), Associative mechanisms in conditioning. Hillsdale, N.J: Erlbaum, 1978.

Moore, J. W. Information processing in space-time by the hippocampus. Physiological Psychology, 1979, 7, 224-232.

Moore, J. W., Goodell, N. A., \& Solomon, P. R. Central cholinergic blockade by scopolamine and habituation, classical conditioning, and latent inhibition of the rabbit's nictitating membrane response. Physiological Psychology, 1976, 4, 395-399.

Moore, J. W., \& Stickney, K. J. Formation of attentionalassociative networks in real time: Role of the hippocampus and implications for conditioning. Physiological Psychology, 1980, 8, 207-217.

Nadel, L., \& Willner, J. Context and conditioning: A place for space. Physiological Psychology, 1980, 8, 218-2.28.

O'KeEFE, J. Place units in the hippocampus of the freely moving rat. Experimental Neurology, 1976, 51, 78-109.

O'KeEFE, J., \& BLACK, A. H. Single unit and lesion experiments on sensory inputs to the hippocampal cognitive map. In Functions of the septo-hippocampal system. CIBA Foundation Symposium 58. New York: Elsevier, 1978.

O'KeEFE, J., \& Dostrovsky, J. The hippocampus as a spatial map. Preliminary evidence from unit activity in the freely moving rat. Brain Research, 1971, 34, 171-175.

O'KeEFE, J., \& NADEL, L. The hippocampus as a cognitive map. London: Oxford University Press, 1978.

O'Keefe, J., Nadel, L., Kieghtly, S., \& Kill, D. Fornix lesions selectively abolish place learning in the rat. Experimental Neurology, 1975, 48, 152-166.

Olton, D. S., Branch, M., \& Best, P. J. Spatial correlates of hippocampal unit activity. Experimental Neurology, 1978, 58, 387-409.

Olton, D. S., Walker, J. A., \& Gage, F. H. Hippocampal connections and spatial discrimination. Brain Research, 1978, 39, 295-308.

Powell, D. A., Mankowski, D., \& Buchanan, S. Concomitant heart rate and corneoretinal potential conditioning in the rabbit (Oryctolagus cuniculus): Effects of caudate lesions. Physiology \& Behavior, 1978, 20, 143-150. 
Powflla, D. A., Mulligan, W. L., \& Buchanan, S. L. Orienting and classical conditioning in the rabbit (Orvctolagus cuniculus): Effect of septal area lesions. Physiology \& Behavior, 1976, 17, 955-962.

POWELL, G. Conditioning of the rabbit's nictitating membrane response with electrical stimulation of the brain as the unconditioned stimulus. Unpublished masters thesis, University of Massachusetts, Amherst, 1980.

PROKASY, W. F. Developments with the two-phase model applied to human eyelid conditioning. In A. H. Black \& W. F. Prokasy (Eds.), Classical conditioning. II. New York: Appleton-CenturyCrofts, 1972.

Rriss, S., \& Wagner, A. R. CS habituation produces a "latent inhibition effect" but no active conditioned inhibition. Learning and Motivation, 1972, 3, 237-245.

Rrscori.A. R. A. Paviovian conditioning and its proper control procedures. Psvchological Revien', 1967, 74, 71-80.

Rr.scorl.A, R. A. Variation in the effectiveness of reinforcement and nonreinforcement following prior inhibitory conditioning. l.e'urning and Motivation, 1971, 2, 113-123.

Salafia, W. R., \& Allan, A. M. Conditioning and latent inhibition with electrical stimulation of hippocampus. Physiological Psychology, 1980, 8, 247-253.

Salafia, W. R., Chiaia, N. L., \& Ramirez, J. J. Retardation of rabbit nictitating membrane response conditioning by subscisure electrical stimulation of hippocampus. Physiology \& Behavior, 1979, 22, 451-455.

Salafia, W. R., Romano, A. G., Tyvan, T. T., \& Host, K. C. Disruption of rabbit (Oryctolagus cuniculus) nictitating membrane conditioning by posttrial electrical stimulation of hippocampus. Physiology \& Behavior, 1977. 18, 207-213.

Schmaltz, L. W., \& Theios. J. Acquisition and extinction of a classically conditioned response in hippocampectomized rabbits (Oryctolagus cuniculus). Journal of Comparative and Physiologicul Psvchologv, 1972, 79, 328-333.

Sмгтн, M. C. Acetylcholine release from the cholinergic septo- hippocampal pathway. Life Sciences, 1974, 14, 2159-2166.

Solomon, P. R. Role of the hippocampus in blocking and conditioned inhibition of the rabbit's nictitating membrane response. Journal of Comparative and Physiological Psychology, 1977, 91, 407-417.

Solomon, P. R. Temporal versus spatial information processing theories of hippocampal function. Psychological Bulletin, 1979, 86, 1272-1279.

Solomon, P. R., Brennan, G., \& Moore, J. W. Latent inhibition of the rabbit's nictitating membrane response as a function of CS intensity. Bulletin of the Psychonomic Society, 1974, 4, 445-448.

Solomon, P. R., Kiney, C. A., \& Scott, D. S. Disruption of latent inhibition following systemic administration of parachlorophenylalanine (PCPA). Physiology \& Behavior, 1978, 26. 265-271.

Solomon, P. R., \& Moore, J. W. Latent inhibition and stimulus generalization of the classically conditioned nictitating membrane response in rabbits (Oryctolagus cuniculus) following dorsal hippocampal ablation. Journal of Comparative and Physiological Psychology, 1975, 89, 1192-1203.

Stumpf, C., Petsche, H., \& Gogolak, G. The significance of the rabbit's septum as a relay station between the midbrain and the hippocampus. II. The differential influence of drugs upon both cell firing pattern and the hippocampus theta activity. Electroencephalography and Clinical Neurophysiology, 1962, 14, 212-219.

Thompson, R. F., Berger, T. W., Cegavske, C. F., Patterson, M. M., Roemer, R. A., Teyler, T. J., \& Young, R. A. The search for the engram. American Psychologist, 1976, 31, 209-227.

Young, R. A., Cegavske, C. F., \& Thompson, R. F. Toneinduced changes in excitability of abducens motoneurons in the reflex path of the rabbit nictitating membrane response. Journal of Comparative and Physiological Psychology, 1976, 90, 424-434. 